\title{
Adaptive Fault Tolerant Stabilization for Uncertain MIMO Systems with Actuation Failures
}

\author{
Wanqin Xiang ${ }^{1}$, Wei $\mathrm{Ao}^{2}$ and Yiyuan $\mathrm{Chen}^{3}$ \\ ${ }^{1,3}$ Chongqing Electric Power College, Chongqing 400028, China \\ ${ }^{2}$ Chongqing Communication College, Chongqing 400035, China \\ malaqincaixwq@163.com,craneaow@163.com,chenyiyuancq@sina.com
}

\begin{abstract}
This paper considers the state stabilization problem for a class of non-affine uncertain MIMO systems with actuation failures. An adaptive robust fault-tolerant control is developed to compensate the affect of uncertainties, actuator failures. And theoretical analysis based on a Lyapunov-like approach demonstrates that, under some proper conditions, the close-loop system would be stabilized and ultimately uniformly bounded state error could be guaranteed by the controller. Further, the proposed controller is no need for on-line fault detection and diagnosis unit, and inexpensive to compute. At last, numerical simulations are provided to validate and illustrate the benefits and effectiveness of the proposed control scheme.
\end{abstract}

Keywords: adaptive control; fault-tolerant; state stabilization; actuator failure

\section{Introduction}

An ideal controller requires an exact model of physical systems to achieve favorable control performance [1]. But it is quit hard for designer to establish an exact model. So there usually exist some uncertainties between models and physical systems. Even more, in most practical control systems, besides the uncertainty, failures (including failures of sensors, actuators, and even the plant itself) may occur at uncertain time and the size of a fault is also unknown[2]. The faults may lead to performance deterioration or even instability of the system. Therefore, research on fault tolerant control (FTC) has received great attention over the past decades. The purpose of FTC is to let the systems operate in safe conditions and with proper performances whenever plant or its components are healthy or faulted. The existing fault-tolerant control approaches can be broadly classified as active FTC (AFTC) and passive FTC (PFTC) [3]. The active FTC requires a fault detection and diagnosis (FDD) to detect and identify the failures on-line, and a control reconfiguration (CR) to reassignment the controller according to the on-line faultdetection information[4]-[5]. The passive approaches exploit the inherent redundancy of the controlled systems, and design a single controller that is robust against the faults and uncertainties [6]-[11]. And remarkable progress have been made in FTC, such as neural networks (NN) and fuzzy systems approximation based method[4], FDD-dependent approach[5], robust adaptive approach[6,7], and sliding mode control (SMC) based approach[8,9], LMI-based approach[10], observer based approach[11], etc.

Our work is motivated by the following three observations. Firstly, the existed AFTC approaches or FDD-dependent approaches are depended heavy online computation. Secondly, the existed FTC approaches including both PFTC and AFTC approaches mostly do not concern the presence of quantization of input and state. Thirdly, the most existed approaches for quantized control are hardly to deal with the problem of actuator failures. 
I this research, we consider the general class of non-affine MIMO systems, which is inherently nonlinear, with model and/or parameter uncertainties, external disturbance, actuator faults, and with quantization for both states and inputs. Moreover, uncertainties may be state-dependent, and the actuator faults are assumed unpredictable during the system operation. And the system considered in the work can be described in Figure 1.

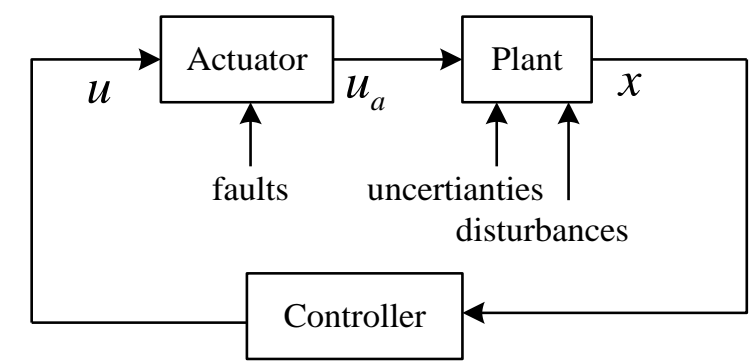

Figure 1. The Block Diagram of the FTC System

( $x$ is the state of the plant, $u$ is the control input computed by the controller, and $u_{a}$ is the actuation signal with failures)

The contributions of this work are threefold: (i) some sufficient conditions are proposed in which the FTC exists for non-affine uncertain MIMO systems under consideration. (ii) An adaptive robust FTC for non-affine uncertain MIMO systems is proposed. (iii) A Lyapunov-like approach is adopted in analysis to guarantee the closeloop system is stable and the state error would be ultimately uniformly bounded, subject to model and parameter uncertainties, disturbances, and actuator faults.

The remainder of this paper is organized as follows. Section II formally states the systems of interest and the control problem. Section III proposes an adaptive control scheme for systems with actuator failures, and along with theorems summarizing the theoretical properties of the controller. Section IV shows numerical simulation, which could demonstrate various effects of the proposed control method. Finally, section V gives some concluding comments to the paper.

\section{Problem Statement}

\subsection{Preliminaries}

Consider a non-affine uncertain MIMO dynamic system described in the following form:

$$
\dot{\mathbf{x}}(t)=\mathbf{F}(\mathbf{x}(t), \mathbf{u}(t), t)+\Delta \mathbf{F}(\mathbf{x}(t), t)
$$

where $\mathbf{x}=\left[x_{1}, x_{2}, \cdots, x_{n}\right]^{T} \in \mathfrak{R}^{n}$ is the state vector, which is assumed to be available for measurement, and this condition is standard for state track; $\mathbf{u}(t)=\left[u_{1}, u_{2}, \cdots, u_{m}\right]^{T} \in \mathfrak{R}^{m}$ is the control input vector; $m$ is the number of system inputs and $m \leq n ; \mathbf{F}=\mathbf{F}(\mathbf{x}, \mathbf{u}, t) \in \mathfrak{R}^{n}$ is a known smooth (or differential) nonlinear vector-valued function; $\Delta \mathbf{F}(\mathbf{x}, t) \in \mathfrak{R}^{n}$ collects all model and parameter uncertainties as well as external disturbance, which is normbounded. Further more, it is normal to assume that the required properties of the existence and uniqueness of solutions of (1) are satisfied. Without loss of generality, $\mathbf{x}=0, \mathbf{u}=0$ is assumed to be the equilibrium point of (1) in the presence of $\Delta \mathbf{F}=0$, and any compact neighborhood of origin $U \subset \mathfrak{R}^{n}$ is to consideration, which could be arbitrarily large.

According to the mean value theorem utilized in [12], $\mathbf{F}$ could be rewritten as follows.

$$
\mathbf{F}(\mathbf{x}, \mathbf{u}, t)=\mathbf{f}(\mathbf{x}, t)+\mathbf{B}\left(\mathbf{x}, \mathbf{u}_{\Lambda}, t\right) \mathbf{u}(t)
$$


where $\mathbf{f}(\mathbf{x}, t)=\left.\mathbf{F}(\mathbf{x}, \mathbf{u}, t)\right|_{\mathbf{u}=0}, \quad \mathbf{B}\left(\mathbf{x}, \mathbf{u}_{\Lambda}, t\right)=\left[b_{i j}\right] \in \mathfrak{R}^{n \times m}, \quad$ and $\quad b_{i j}=\partial F_{i}(\mathbf{x}, \mathbf{u}, t) /\left.\partial u_{j}\right|_{u_{j}=u_{\wedge i j}}$, $\mathbf{u}_{\Lambda}=\left[u_{\Lambda 1}, \cdots, u_{\Lambda j}, \cdots u_{\Lambda m}\right], \quad u_{\Lambda j}=\left[u_{\Lambda 1 j}, \cdots, u_{\Lambda i j}, \cdots u_{\Lambda m j}\right], u_{\Lambda i j}=\lambda_{i j} u_{j}, \lambda_{i j} \in[0,1]$, and $\lambda_{i j}$ is a unknown positive scalar, $i=1, \cdots, n, j=1, \cdots, m$. Then, the dynamics could be rewritten as follows.

$$
\dot{\mathbf{x}}=\mathbf{f}(\mathbf{x}, t)+\mathbf{B}\left(\mathbf{x}, \mathbf{u}_{\Lambda}, t\right) \mathbf{u}(t)+\mathbf{o}(\mathbf{x}, \mathbf{t})
$$

where $\mathbf{o}(\mathbf{x}, \mathbf{t})=\Delta \mathbf{F}(\mathbf{x}, \mathbf{t})$, and $\mathbf{o}(\mathbf{x}, \mathbf{t}), \mathbf{B}\left(\mathbf{x}, \mathbf{u}_{\Lambda}, t\right)$ are both unknown.

The control objective is to design an adaptive robust control scheme, such that the system state $\mathbf{x}(t)$ can be stabled, which means it would asymptotically stable or in a very neighbor of origin.

For the dynamic system under consideration, we would introduce some reasonable assumptions to achieve the control objective.

Under Assumption 1 and 2, the dynamic system (3) can be expressed as follows.

$$
\dot{\mathbf{x}}(t)=\mathbf{B}_{0}(\mathbf{I}+\mathbf{H}) \mathbf{u}+\xi(\mathbf{x}, t)
$$

where $\mathbf{B}_{0}, \mathbf{H}$ and $\xi(\mathbf{x}, t)$ are defined in Assumption 1 and 2 , and $\xi(\mathbf{x}, t)=\mathbf{f}(\mathbf{x}, t)+\mathbf{o}(\mathbf{x}, \mathbf{t})$. Further, conduct full-rank factorization of $\mathbf{B}_{0}$. As $\operatorname{rank}\left(\mathbf{B}_{0}\right)=r$, it leads to $\mathbf{B}_{0}=\mathbf{C}_{1} \mathbf{L}_{1}$, where $\mathbf{C}_{1} \in \mathfrak{R}^{n \times r}, \mathbf{L}_{1} \in \mathfrak{R}^{r \times m}$ and $\operatorname{rank}\left(\mathbf{C}_{1}\right)=\operatorname{rank}\left(\mathbf{L}_{1}\right)=r$.

\section{Assumption 1}

There exists a known norm-bounded smooth matrix $\mathbf{B}_{0} \square \mathbf{B}_{0}(\mathbf{x}, t)$, and an unknown norm-bounded smooth matrix $\mathbf{H} \square \mathbf{H}\left(\mathbf{x}, \mathbf{u}_{\Lambda}, t\right)$, such that, $\mathbf{B}\left(\mathbf{x}, \mathbf{u}_{\Lambda}, t\right)=\mathbf{B}_{0}(\mathbf{I}+\mathbf{H})$, where $\mathbf{B}_{0} \in \mathfrak{R}^{n \times m}, \mathbf{H} \in \mathfrak{R}^{m \times m}$. And the partial differential $\partial \mathbf{B}_{0}(\mathbf{x}, t) / \partial \mathbf{x}$ is norm-bounded.

\section{Assumption 2}

Denotes $\boldsymbol{v}=\boldsymbol{v}(\mathbf{x}, t) \square \partial V_{1}(\mathbf{x}) / \partial \mathbf{x}, \boldsymbol{\rho}^{T}=\boldsymbol{\rho}(\mathbf{x}, t)^{T} \square \boldsymbol{v}^{T} \mathbf{C}_{1}, \boldsymbol{\sigma}^{T}=\boldsymbol{\sigma}(\mathbf{x}, t)^{T} \square \boldsymbol{v}^{T} \mathbf{B}_{0}$ and $\boldsymbol{v} \in \mathfrak{R}^{n}$, $\boldsymbol{\rho} \in \mathfrak{R}^{r}$. There exists some trajectories $\mathbf{x}$, such that, $\boldsymbol{\rho}=0$ holds, if and only if $\mathbf{x}=0$.

\section{Assumption 3}

For all $\mathbf{P}(t)$ belonged to allowed fault mode, the controllability of the system still holds, that is equivalent that, $\operatorname{rank}\left(\mathbf{B}_{0}\right)=\operatorname{rank}\left(\mathbf{B}_{0} \mathbf{P}\right)=r$, for all $\mathbf{P} \in \Delta_{\mathbf{P}^{j}}, j=1, \cdots, L$, where $\mathbf{P}(t)$ is defined in subsection 3 .

\section{Remark 1}

Under Assumption 1, there exists an unknown non-negative scalar $0 \leq \bar{\lambda}<L_{\infty}$, such that $\|\mathbf{H}\|<\bar{\lambda}$. Further there exists an unknown scalar $\underline{\lambda} \in(0,1)$, such that $\mathbf{x}^{T}(\underline{\lambda} \mathbf{I}+\mathbf{H}) \mathbf{x} \geq 0$, for $\forall \mathbf{x} \in \mathfrak{R}^{n}$, so that control direction of the dynamic system is certain. Notice that, as $\mathbf{I}+\mathbf{H}=(1-\underline{\lambda}) \mathbf{I}+(\underline{\lambda} \mathbf{I}+\mathbf{H})$, it is easy to get $\mathbf{x}^{T}(\mathbf{I}+\mathbf{H}) \mathbf{x} \geq(1-\underline{\lambda})\|\mathbf{x}\|^{2}$, for $\forall \mathbf{x} \in \mathfrak{R}^{n}$.

\section{Remark 2}

Under Assumption 2, and due to the definition of $\boldsymbol{v}, \boldsymbol{v}=0$ would hold if and only if $\mathbf{x}=0$. So we can define $\mu(\mathbf{x})=\|\mathbf{v}(\mathbf{x})\| /\|\mathbf{x}\|$ as $\mathbf{x} \neq 0$, and $\mu(\mathbf{x})$ is an unknown positive function, whose upper bounds is $\bar{\mu}_{m}$, and $0<\bar{\mu}_{m}<L_{\infty}$, then for all $\mathbf{x},\|\mathbf{v}(\mathbf{x})\| \leq \bar{\mu}_{m}\|\mathbf{x}\|$. Actually, $\bar{\mu}_{m}$ is an unknown positive constant scalar. Further more, it is noted that, $\bar{\mu}_{m}$ will not be used in the controller design, but just for the analysis.

Further, denotes $s=s(\mathbf{x}, t) \square\|\boldsymbol{\rho}\| /\|\mathbf{x}\|$, then $\|\boldsymbol{\rho}\|=s \cdot\|\mathbf{x}\|$, and $s(\mathbf{x}, t)$ is an unknown positive function. Under Assumption 2, $s$ is positive for all $\mathbf{x} \neq 0$ and $\mathbf{B}_{0}$. Let $s_{m}$ be the lower 
bound of $s$, and it is easy to get that $0<s_{m}<L_{\infty}$, which means $\|\boldsymbol{\rho}\|=s \cdot\|\mathbf{x}\| \geq s_{m}\|\mathbf{x}\|$, and then $\|\mathbf{x}\| \leq s_{m}{ }^{-1}\|\boldsymbol{\rho}\|$. Similar to $\bar{\mu}_{m}$, there is no need to determine the exact information of $s_{m}$.

It is noted that, the full-rank factorization of $\mathbf{B}_{0}$ is not used in the control design, but just for analysis. And in actually, $\boldsymbol{\rho}$ is a virtual signal which is not need to measured or computed, but just to assist the theoretical analysis.

\section{Remark 3}

The existence and uniqueness of the solution of (2) in $U$ is equal to that the dynamic system satisfied the Lipschitz condition [30], which means $\|\mathbf{f}(\mathbf{x}, t)\| \leq L_{1}\|\mathbf{x}\|$, where $L_{1}<L_{\infty}$ is a unknown positive constant. As the fact that $\Delta \mathbf{F}(\mathbf{x}, t)$ is normbounded, and with existence and uniqueness of solution to the dynamical system under consideration, one could obtain that, there exists an unknown positive scalar $\delta \square \delta(\mathbf{x}, t)$ and $\delta<L_{\infty}$, such that $\|\xi(\mathbf{x}, t)\| \leq \bar{\mu}_{m}^{-1} s_{m} \delta \Phi$, where $\bar{\mu}_{m}$ and $s_{m}$ are defined as before, and $\Phi=1+\|\mathbf{x}\|$.

\subsection{Actuation Failures}

In this work, actuation failures considered include actuator outage, loss of effectiveness and stuck. Let $u_{a i j}$ represents the real signal from the $i$ th actuator that has failed in the $j$ th fault mode. Then, we describe the following fault modes:

$$
u_{a i j}=p_{i}^{j} u_{i}(t)+u_{s i}(t)
$$

where $i=1, \cdots, m, j=1, \cdots, L, p_{i}^{j}$. The index $j$ denotes the $j$ th fault mode and $L$ is the total fault modes. For every fault mode, $\underline{p}_{i}^{j}$ and $\bar{p}_{i}^{j}$ represent the unknown lower and upper bounds of $p_{i}^{j}$, respectively. $u_{s i}(t)$ is the unparameterizable bounded time-varying portion of actuation failures in the $i$ th actuator. Following the practical case, we have $0 \leq p_{i}^{j} \leq p_{i}^{j} \leq \bar{p}_{i}^{j} \leq 1$. And in many literatures, $p_{i}^{j}$ is so-called health indicator.

Denotes $\quad \mathbf{u}_{a j}=\left[u_{a 1 j}(t), \cdots, u_{a m j}(t)\right]^{T}=\mathbf{P}^{j} \mathbf{u}_{i n}(t)+\mathbf{u}_{s}(t), \quad$ where $\quad \mathbf{u}_{i n}(t)=\left[u_{1}(t), \cdots, u_{m}(t)\right]^{T} ，$ $\mathbf{u}_{s}(t)=\left[u_{s 1}(t), \cdots, u_{s m}(t)\right]^{T}, \mathbf{P}^{j}=\operatorname{diag}\left\{p_{1}^{j}, \cdots, p_{m}^{j}\right\}, p_{i}^{j} \in\left[\underline{p}_{i}^{j}, \bar{p}_{i}^{j}\right]$, and $u_{i}(t)$ is the $i$ th input to be designed, $i=1, \cdots, m, \quad j=1, \cdots, L$.Then the sets with above structure can be defined by

$$
\Delta_{\mathbf{P}^{j}}=\left\{\mathbf{P}^{j} \mid \mathbf{P}^{j}=\operatorname{diag}\left\{p_{1}^{j}, \cdots, p_{m}^{j}\right\}, p_{i}^{j} \in\left[\underline{p}_{i}^{j}, \bar{p}_{i}^{j}\right]\right\} .
$$

For convenience in the following sections, for all possible fault modes $L$, the following uniform actuator fault mode is exploited:

$$
\mathbf{u}_{a}(t)=\mathbf{P} \mathbf{u}_{i n}(t)+\mathbf{u}_{s}(t)
$$

where $\mathbf{P} \in \Delta_{\mathbf{p}^{j}}$. It is noted that, under the actuation failures, $\mathbf{u}_{a}(t)$ instead of $\mathbf{u}(t)$ should be used in the dynamic system (4).

In order to ensure the fault-tolerant objective under the actuation failures, the Assumption 4 in the FTC design also should be valid.

\subsection{Control Objectives}

The control objective in this paper is as follows.

(i) find some sufficient conditions which could ensure the existence of the FTC for the non-affine uncertain systems with actuation failures and quantization under consideration;

(ii) design a suitable adaptive robust control to compensate both the quantizer effects and actuator faults;

(ii) propose an approach to guarantee the convergence of the proposed adaptive robust FTC.

\section{Control Scheme}


In this section, we would present some results for the non-affine uncertain systems under consideration.

Under the actuation failures in form of (6), the dynamic system (4) can be expressed as

$$
\dot{\mathbf{x}}(t)=\mathbf{B}_{0}(\mathbf{I}+\mathbf{H})\left(\mathbf{P} \mathbf{u}_{i n}(t)+\mathbf{u}_{s}(t)\right)+\xi^{\prime}(\mathbf{x}, t)
$$

where $\xi^{\prime}(\mathbf{x}, t)=\mathbf{B}_{0}(\mathbf{I}+\mathbf{H}) \mathbf{u}_{s}(t)+\xi(\mathbf{x}, t)$.

Further, we would introduce a proposition, which is used in the theoretical analysis.

\section{Proposition 1}

Consider the error dynamic equation (7) with Assumption 1, 2 and 3. If one of the following conditions is satisfied, there exists an unknown positive constant $0<b_{\mathrm{m}}<L_{\infty}$, such that $\boldsymbol{\rho}^{T} \mathbf{L}_{1}(\mathbf{I}+\mathbf{H}) \mathbf{P} \mathbf{L}_{1}^{T} \boldsymbol{\rho} \geq b_{m}\|\boldsymbol{\rho}\|^{2}$, for $\forall \boldsymbol{\rho} \in \mathfrak{R}^{r}$.

1) $\mathbf{H}=0$;

2) $\mathbf{H} \neq 0$, and $\mathbf{H}$ is symmetry;

3) $\mathbf{H} \neq 0$, and $\mathbf{H}$ is not symmetry, while $\Gamma_{1}$ is symmetry and non-negative, where $\Gamma_{1}=\left[\mathbf{P}_{1}+\mathbf{P}_{1}^{T}\right] / 2$ and $\mathbf{P}_{1}=(\mathbf{I}+\mathbf{H}) \mathbf{P}$.

\section{Proof}

1) when $\mathbf{H}=0$, we have $\mathbf{I}+\mathbf{H}=\mathbf{I}$

Conducts full-rank factorization of $\mathbf{P}$. Note that $\mathbf{P} \in \mathfrak{R}^{m \times m}$ is diagonal, which means $\mathbf{P}=\mathbf{C}_{2} \mathbf{C}_{2}^{T}$, where $\mathbf{C}_{2} \in \mathfrak{R}^{m \times r^{\prime}}$ is diagonal, and as under Assumption $4, \operatorname{rank}\left(\mathbf{B}_{0} \mathbf{P}\right)=r$, then it is easy to get $\operatorname{rank}\left(\mathbf{C}_{2}\right)=r^{\prime}, r \leq r^{\prime} \leq m$.

Further more, as $\mathbf{B}_{0}=\mathbf{C}_{1} \mathbf{L}_{1}$, so one can obtain $\operatorname{rank}\left(\mathbf{C}_{1} \mathbf{L}_{1} \mathbf{C}_{2} \mathbf{C}_{2}^{T}\right)=r$, and $\mathbf{L}_{1} \mathbf{C}_{2} \in \Re^{r \times r^{\prime}}$, it could lead to $\operatorname{rank}\left(\mathbf{L}_{1} \mathbf{C}_{2}\right)=r$, which means $\mathbf{L}_{1} \mathbf{C}_{2}$ is row full rank. And as $\mathbf{L}_{1} \mathbf{C}_{2}$ is row full rank, then $\mathbf{L}_{1} \mathbf{C}_{2} \mathbf{C}_{2}^{T} \mathbf{L}_{1}^{T} \in \Re^{r \times r}$ is symmetry and positive. It is equivalently that, $\boldsymbol{\rho}^{T} \mathbf{L}_{1} \mathbf{C}_{2} \mathbf{C}_{2}^{T} \mathbf{L}_{1}^{T} \boldsymbol{\rho}>0$, for $\forall \boldsymbol{\rho} \neq 0$.

Then one can find a positive constant $b_{\mathrm{m}}$, such that, $\boldsymbol{\rho}^{T} \mathbf{L}_{1} \mathbf{P} \mathbf{L}_{1}^{T} \boldsymbol{\rho}=\boldsymbol{\rho}^{T} \mathbf{L}_{1} \mathbf{C}_{2} \mathbf{C}_{2}^{T} \mathbf{L}_{1}^{T} \boldsymbol{\rho} \geq b_{m}\|\boldsymbol{\rho}\|^{2}$, for $\forall \boldsymbol{\rho} \in \mathfrak{R}^{r}$.

Therefore, the proposition holds when $\mathbf{H}=0$.

2) when $\mathbf{H} \neq 0$, and $\mathbf{H}$ is symmetry, $\mathbf{I}+\mathbf{H}$ is symmetry.

According to Assumption 1, $\mathbf{x}^{T}(\mathbf{I}+\mathbf{H}) \mathbf{x} \geq\left(1-\underline{\lambda} \| \mathbb{Z}^{2}\right.$, for $\forall \mathbf{x} \in \mathfrak{R}^{n}$, and $0<\underline{\lambda}<1$. And as the condition, $\mathbf{I}+\mathbf{H}$ is symmetry, so, one could conclude that, $\mathbf{I}+\mathbf{H}$ is symmetry and positive definite. Denotes $\mathbf{P}_{1}=(\mathbf{I}+\mathbf{H}) \mathbf{P}$, and as the definition of $\mathbf{P}, \mathbf{P}$ is symmetry and non-negative, further more, it is easy to obtain that, $\mathbf{P}_{1}$ is symmetry and non-negative.

Similar to the previous proof, for the $\boldsymbol{\rho} \neq 0$, as $\mathbf{P}_{1}$ is symmetry and non-negative, there exists an unknown positive constant $b_{\mathrm{m}}$, such that, $\boldsymbol{\rho}^{T} \mathbf{L}_{1}(\mathbf{I}+\mathbf{H}) \mathbf{P} \mathbf{L}_{1}^{T} \boldsymbol{\rho} \geq b_{m}\|\boldsymbol{\rho}\|^{2}$, for $\forall \boldsymbol{\rho} \in \Re^{r}$.

3) when $\mathbf{H} \neq 0, \mathbf{H}$ is not symmetry, and $\Gamma_{1}$ is symmetry and non-negative

As $\mathbf{P}_{1}=(\mathbf{I}+\mathbf{H}) \mathbf{P}$, one could obtain that $\Gamma_{1}=\left[\mathbf{P}_{1}+\mathbf{P}_{1}^{T}\right] / 2, \Gamma_{2}=\left[\mathbf{P}_{1}-\mathbf{P}_{1}^{T}\right] / 2$, where $\Gamma_{1}$ is symmetry and non-negative, and $\Gamma_{2}$ is skew symmetry, which means, for $\forall \boldsymbol{\rho} \in \mathfrak{R}^{r}$, $\boldsymbol{\rho}^{T} \mathbf{L}_{1} \Gamma_{1} \mathbf{L}_{1}^{T} \boldsymbol{\rho} \geq 0, \boldsymbol{\rho}^{T} \mathbf{L}_{1} \Gamma_{2} \mathbf{L}_{1}^{T} \boldsymbol{\rho}=0$.

And $\boldsymbol{\rho}^{T} \mathbf{L}_{1}(\mathbf{I}+\mathbf{H}) \mathbf{P} \mathbf{L}_{1}^{T} \boldsymbol{\rho}=\boldsymbol{\rho}^{T} \mathbf{L}_{1} \Gamma_{1} \mathbf{L}_{1}^{T} \boldsymbol{\rho}+\boldsymbol{\rho}^{T} \mathbf{L}_{1} \Gamma_{2} \mathbf{L}_{1}^{T} \boldsymbol{\rho}$, so that, one could get, $\boldsymbol{\rho}^{T} \mathbf{L}_{1}(\mathbf{I}+\mathbf{H}) \mathbf{P} \mathbf{L}_{1}^{T} \boldsymbol{\rho}=\boldsymbol{\rho}^{T} \mathbf{L}_{1} \Gamma_{1} \mathbf{L}_{1}^{T} \boldsymbol{\rho} \geq 0$.

As $\Gamma_{1}$ is symmetry and non-negative, let use $\Gamma_{1}$ in the place of $\mathbf{P}$, and similar to the previous proof, the results may be reached in this case. 
It is equivalently that, one could find a positive constant $b_{\mathrm{m}}$, such that, $\boldsymbol{\rho}^{T} \mathbf{L}_{1}(\mathbf{I}+\mathbf{H}) \mathbf{P} \mathbf{L}_{1}^{T} \boldsymbol{\rho}=\boldsymbol{\rho}^{T} \mathbf{L}_{1} \Gamma_{1} \mathbf{L}_{1}^{T} \boldsymbol{\rho} \geq b_{m}\|\boldsymbol{\rho}\|^{2}$, for $\forall \boldsymbol{\rho} \in \mathfrak{R}^{r}$.

\section{Remark 4}

Proposition 1 proposes some critical rules to verify the control direction of the faulttolerant control problem of non-affine uncertain system under consideration. It is noted that, these conditions are sufficient, and the proposition could ensure that the control direction is maintained, if these conditions are satisfied.

According to the definition of $\boldsymbol{\rho}$ and $\boldsymbol{\sigma}$, one could get $\boldsymbol{\rho}^{T} \mathbf{L}_{1}=\boldsymbol{\sigma}^{T}$, further, as $\mathbf{L}_{1}$ is row full rank, it is easy to get that $\boldsymbol{\sigma} \neq 0$ while $\boldsymbol{\rho} \neq 0$, which means $\boldsymbol{\sigma}^{T}(\mathbf{I}+\mathbf{H}) \mathbf{P} \boldsymbol{\sigma}=\boldsymbol{\rho}^{T} \mathbf{L}_{1}(\mathbf{I}+\mathbf{H}) \mathbf{P} \mathbf{L}_{1}^{T} \boldsymbol{\rho}$. Therefore, there exists an unknown positive constant $0<p_{\mathrm{m}}<L_{\infty}$, such that $\boldsymbol{\sigma}^{T}(\mathbf{I}+\mathbf{H}) \mathbf{P} \boldsymbol{\sigma} \geq b_{m}\|\boldsymbol{\rho}\|^{2}=p_{m}\|\boldsymbol{\sigma}\|^{2}$.

\section{Remark 5}

As $\mathbf{u}_{s}(t)$ and $\xi(\mathbf{x}, t)$ are both norm-bounded, it means that, there exists an unknown positive scalar $\delta^{\prime} \square \delta^{\prime}(\mathbf{x}, t)$ and $\delta^{\prime}<L_{\infty}$, such that $\left\|\xi^{\prime}(\mathbf{x}, \mathbf{t})\right\| \leq \bar{\mu}_{m}^{-1} s_{m} \Phi \delta^{\prime}$, where $\bar{\mu}_{m}$ and $s_{m}$ are defined as before, and $\Phi=1+\|\mathbf{x}\|$.

\subsection{Adaptive Robust FTC Design Under Actuation Failures}

Under proposition 1, an adaptive robust fault tolerant control for dynamic system (7) is designed as follows.

$$
\mathbf{u}_{i n}=-k(t) \Phi \hat{\delta} \boldsymbol{\sigma}-k_{0} \boldsymbol{\sigma}
$$

where $k(t)=k_{1} /\|\boldsymbol{\sigma}\|+\varepsilon, k_{0}>0$, and $\hat{\delta}$ is the estimation of $\delta^{\prime}$, and $\boldsymbol{\sigma}$ defined as before. It is noted that, the designed control law consists of two parts, $-k(t) \Phi \hat{\delta} \boldsymbol{\sigma}$ is the adaptive control, and $-k_{0} \boldsymbol{\sigma}$ is the robust control.

And the parameter update law is implemented as follows.

$$
\dot{\hat{\delta}}=-a_{1} \hat{\delta}+a_{2} \Phi \frac{\|\boldsymbol{\sigma}\|^{2}}{\|\boldsymbol{\sigma}\|+\varepsilon}
$$

where $a_{1}, a_{2}$ are some designed parameters, and $\varepsilon=\tau / 1+\Phi, \Phi=1+\|\mathbf{x}\|$, and $\tau$ is a positive scalar that can be arbitrarily small.

\section{Stability Analysis}

\section{Theorem 1}

Consider the closed-loop adaptive system consisting of dynamic equation (7) with actuation failures, the controller (8), the parameter update law (9), and under Assumption $1-3$, and Proposition 1 . The state $\mathbf{x}$ could be ensured to stable and ultimately uniformly bounded (UUB), and $\mathbf{x}$ could be made small enough by choosing proper $k_{1}$ and $\tau$. Meanwhile, $\mathbf{x}$ could be made sufficiently small by selecting $k_{1}$ sufficiently large and $\tau$ sufficiently small.

\section{Proof}

Define Lyapunov function $V=V_{1}(\mathbf{x})+V_{2}$, where $V_{1}(\mathbf{x})$ is given in Assumption 1, and $V_{2}=\left(\delta^{\prime}-k \hat{\delta}\right) / 2 k a_{2}, k=k_{1} p_{m}$.

\section{Part A: Derivative of $V$}


Note that $V=V_{1}+V_{2}, \dot{V}=\dot{V}_{1}+\dot{V}_{2}$ and $\boldsymbol{\sigma}^{T}=\left(\partial V_{1}(\mathbf{x}) / \partial \mathbf{x}^{T}\right) \mathbf{B}_{0}$.

$$
\dot{V}_{1}=\frac{\partial V_{1}(\mathbf{x})}{\partial \mathbf{x}^{T}} \dot{\mathbf{x}}=\frac{\partial V_{1}(\mathbf{x})}{\partial \mathbf{x}^{T}}\left\{\mathbf{g}+\mathbf{B}_{0}(\mathbf{I}+\mathbf{H}) \mathbf{P} \mathbf{u}_{i n}+\xi^{\prime}\right\}
$$

Define

$$
\begin{aligned}
& \dot{V}_{11}=\frac{\partial V_{1}(\mathbf{x})}{\partial \mathbf{x}^{T}} \mathbf{g} \leq-\gamma(\|\mathbf{x}\|) \\
& \dot{V}_{12}=\boldsymbol{\sigma}^{T}(\mathbf{I}+\mathbf{H}) \mathbf{P} \mathbf{u}_{i n}=-\hat{\delta} k_{1} p_{m} \frac{\Phi\|\boldsymbol{\sigma}\|^{2}}{\|\boldsymbol{\sigma}\|+\varepsilon}-k_{0} p_{m}\|\boldsymbol{\sigma}\|^{2} \\
& \dot{V}_{13}=\frac{\partial V_{1}(\mathbf{x})}{\partial \mathbf{x}^{T}} \xi^{\prime} \leq \bar{\mu}_{m} s_{m}^{-1}\|\mathbf{e}\| \cdot \bar{\mu}_{m}^{-1} s_{m} \Phi\|\boldsymbol{\sigma}\| \leq \delta^{\prime} \Phi\|\boldsymbol{\sigma}\|
\end{aligned}
$$

And (13) can be rewritten as

$$
\dot{V}_{13} \leq \delta^{\prime} \Phi\|\boldsymbol{\sigma}\|=\delta^{\prime} \Phi\|\boldsymbol{\sigma}\| \frac{\|\boldsymbol{\sigma}\|+\varepsilon}{\|\boldsymbol{\sigma}\|+\varepsilon} \leq \delta^{\prime} \Phi \frac{\|\boldsymbol{\sigma}\|^{2}}{\|\boldsymbol{\sigma}\|+\varepsilon}+\delta^{\prime} \Phi \varepsilon
$$

Note that $\varepsilon=\frac{\tau}{1+\Phi}$, then $\delta^{\prime} \Phi \varepsilon<\delta^{\prime} \tau$.

As $\dot{V}_{2}=\left(\delta^{\prime}-k \hat{\delta}\right)\left(-\frac{1}{a_{2}} \dot{\hat{\delta}}\right)$, it would got that from (10)-(14).

$$
\dot{V}=\dot{V}_{1}+\dot{V}_{2} \leq-\gamma(\|\mathbf{e}\|)-k_{0} p_{m}\|\boldsymbol{\sigma}\|^{2}+\dot{V}^{\prime}
$$

where

$$
\begin{aligned}
\dot{V}^{\prime} & =-\hat{\delta} \Phi k_{1} p_{m} \frac{\|\boldsymbol{\sigma}\|^{2}}{\|\boldsymbol{\sigma}\|+\varepsilon}+\delta \Phi \frac{\|\boldsymbol{\sigma}\|^{2}}{\|\boldsymbol{\sigma}\|+\varepsilon}+\delta^{\prime} \Phi \varepsilon+\left(\delta^{\prime}-k \hat{\delta}\right)\left(-\frac{\dot{\hat{\delta}}}{a_{2}}\right) \\
& =\frac{\|\boldsymbol{\sigma}\|^{2}}{\|\boldsymbol{\sigma}\|+\varepsilon} \Phi\left[\delta^{\prime}-\hat{\delta} k\right]+\left(\delta^{\prime}-k \hat{\delta}\right)\left(-\frac{\dot{\hat{\delta}}}{a_{2}}\right)+\delta^{\prime} \tau
\end{aligned}
$$

Then, the previous equation could be rewritten as follows.

$$
\dot{V}^{\prime}=\frac{1}{a_{2}}\left(\delta^{\prime}-\hat{\delta} k\right)\left[-\dot{\hat{\delta}}+a_{2} \frac{\|\boldsymbol{\sigma}\|^{2}}{\|\boldsymbol{\sigma}\|+\varepsilon} \Phi\right]+\delta^{\prime} \tau
$$

As $\dot{\hat{\delta}}=-a_{1} \hat{\delta}+a_{2} \frac{\|\boldsymbol{\sigma}\|^{2}}{\|\boldsymbol{\sigma}\|+\varepsilon} \Phi$, and (16) can be rewritten.

$$
\dot{V}^{\prime}=\delta^{\prime} \tau-\frac{a_{1}}{a_{2}} k\left(\hat{\delta}-\frac{\delta^{\prime}}{2 k}\right)^{2}+\frac{a_{1}}{4 k a_{2}} \delta^{\prime 2} \leq \delta^{\prime} \tau+\frac{a_{1}}{4 k a_{2}} \delta^{\prime 2}
$$

Insert (17) into (15), then it leads to

$$
\dot{V} \leq-\gamma(\|\mathbf{e}\|)-k_{0} p_{m}\|\boldsymbol{\sigma}\|^{2}+\varepsilon_{0}
$$

where $\varepsilon_{0}=\delta^{\prime} \tau+\frac{a_{1}}{4 k a_{2}} \delta^{\prime 2}=\delta^{\prime} \tau+\frac{a_{1}}{4 k_{1} p_{m} a_{2}} \delta^{\prime 2}$.

\section{Part B: Ultimately Uniformly Bounded of $x$}

As $\gamma(\|\mathbf{x}\|) \geq\|\mathbf{x}\|^{2}$, then $\dot{V} \leq-\|\mathbf{x}\|^{2}+\varepsilon_{0}$. When $\mathbf{x}$ locate outside the neighbor of original, $\Omega=\left\{\mathbf{x} \mid\|\mathbf{x}\| \leq \sqrt{\varepsilon_{0}}\right\}$, it is obtained that $\dot{V}<0$, which means $\mathbf{x}$ will be attracted in to $\Omega$, and cannot go out of it. That is equivalently that, the states are confined in $\Omega$. Furthermore, as $\varepsilon_{0}=\delta^{\prime} \tau+a_{1} \delta^{\prime 2} /\left(4 k_{1} p_{m} a_{2}\right)$, it implies that larger $k_{1}, a_{2}$, and smaller $a_{1}$ and smaller $\tau$ lead to better performance. And the state $\mathbf{x}$ can be made sufficiently small by selecting $k_{1}$ sufficiently large enough, and $\tau$ small enough.

Thus the result stated in Theorem 1 is established. 


\section{Illustrative Example}

Consider the following non-affine uncertain MIMO dynamic system $\dot{\mathbf{x}}=\mathbf{F}(\mathbf{x}, \mathbf{u}, t)+\Delta \mathbf{F}(\mathbf{x}, t)$

$\mathbf{F}=\left[\begin{array}{ll}f_{1} & f_{2}\end{array}\right]^{T}, F_{1}=\left(0.1+u_{1}+0.1 \sin u_{1}\right)\left(3+2 \sin x_{1}\right)+\left(u_{2}+0.2 \sin u_{2}\right) \sin x_{2}-x_{1}$,

$F_{2}=\left(0.1+u_{1}+0.1 \sin u_{1}\right)\left(3+2 \sin x_{2}\right)+\left(u_{2}+0.2 \sin u_{2}\right) \sin x_{1}-x_{2}$,

$\Delta \mathbf{F}=\left[\begin{array}{l}0.1 \sin \left(x_{1}\right)+0.3 \sin (\ln (t+1)) \\ 0.1 \sin \left(x_{2}\right)+0.3 \sin (\ln (t+1))\end{array}\right]$

where $\mathbf{x}=\left[x_{1}, x_{2}\right]^{T}$ is the state vector, and $\mathbf{u}=\left[u_{1}, u_{2}\right]^{T}$ is the input to be designed.

We can get $\mathbf{f}=\left.\mathbf{F}(\mathbf{x}, \mathbf{u}, t)\right|_{\mathbf{u}=0}, \mathbf{B}=\partial \mathbf{F}(\mathbf{x}, \mathbf{u}, t) /\left.\partial \mathbf{u}^{T}\right|_{\mathbf{u}=\mathbf{u}_{\wedge}}$,

$$
\mathbf{f}=\left[\begin{array}{l}
0.3+0.2 \sin x_{1}-x_{1} \\
0.3+0.1 \sin x_{2}-x_{2}
\end{array}\right], \quad \mathbf{B}=\left[\begin{array}{cc}
\left(1+0.1 \cos u_{1 \Lambda}\right)\left(3+2 \sin x_{1}\right) & \left(1+0.2 \cos u_{2 \Lambda}\right) \sin x_{2} \\
\left(1+0.1 \cos u_{1 \Lambda}\right)\left(3+\sin x_{2}\right) & \left(1+0.2 \cos u_{2 \Lambda}\right) \sin x_{1}
\end{array}\right]
$$

where $u_{1 \Lambda}, u_{2 \Lambda}$ are unknown scalars. Then we can get $\mathbf{B}_{0}=\left[\begin{array}{cc}3+2 \sin x_{1} & \sin x_{2} \\ 3+\sin x_{2} & \sin x_{1}\end{array}\right]$, and $\mathbf{H}=\left[\begin{array}{cc}0.1 \cos u_{1 \Lambda} & 0 \\ 0 & 0.2 \cos u_{2 \Lambda}\end{array}\right]$, further, $\mathbf{I}+\mathbf{H}=\left[\begin{array}{cc}1+0.1 \cos u_{1 \Lambda} & 0 \\ 0 & 1+0.2 \cos u_{2 \Lambda}\end{array}\right]$.

Let choose $V_{1}$ as $V_{1}=\frac{1}{2} x_{1}^{2}+\frac{1}{2} x_{2}^{2}+\frac{1}{100} x_{1}^{4}+\frac{1}{100} x_{2}^{4}$, and $\mathbf{g}(\mathbf{x}, t)=\left[\begin{array}{cc}-1 & 0 \\ 0 & -1\end{array}\right]\left[\begin{array}{l}x_{1} \\ x_{2}\end{array}\right]$.

The health indicators in the inputs could be described as follows.

$$
\rho_{1}(t)=\left\{\begin{array}{cc}
1 & 0 \leq t<0.5 \\
0.4 t^{2}-0.6 t+0.5 & 0.5 \leq t<1.5 \\
0.6+0.25 \sin (t) & 1.5 \leq t
\end{array} \quad \rho_{2}(t)=\left\{\begin{array}{cc}
1 & 0 \leq t<0.5 \\
0.25+0.1 \cos (\pi t) & 0.5 \leq t<1.0 \\
0 & 1.0 \leq t
\end{array}\right.\right.
$$

Note that, the second input would be totally outage in a very short time. Figure 2 shows the

health indicators in the input. And the failures in form of uncontrollable portion could be described as: $u_{s 1}(t)=0.1 \sin x_{1}+0.2 \sin (\ln (t+1)), u_{s 2}(t)=0.1 \sin x_{2}+0.2 \sin (\ln (t+1))$.
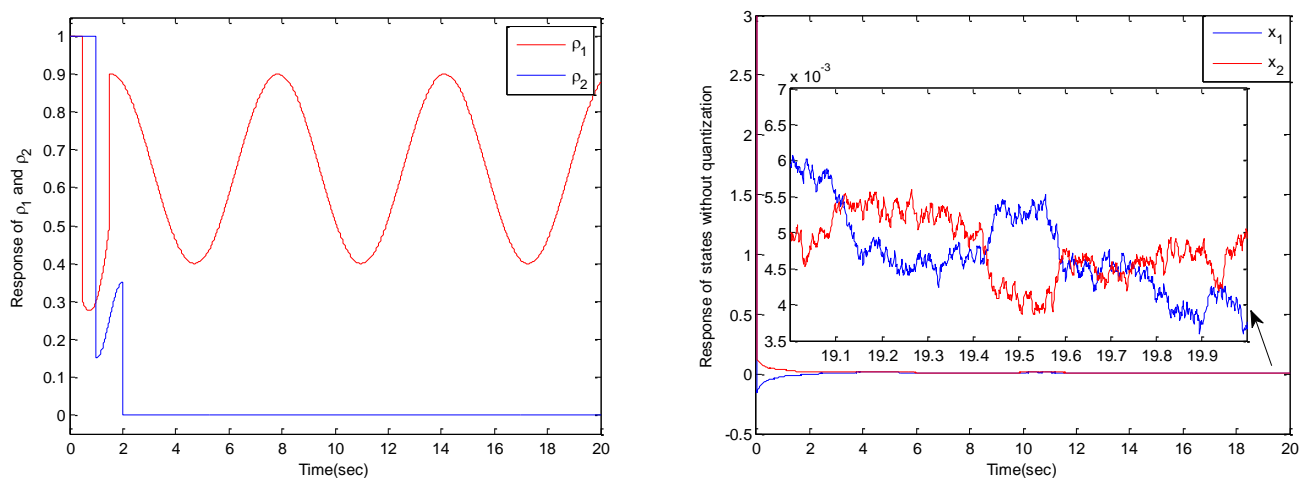

Figure 2. The Health Indicator in the Input Figure 3. Response of State

Notice that, $\operatorname{rank}\left(\mathbf{B}_{0}\right)=\operatorname{rank}\left(\mathbf{B}_{0} \mathbf{P}\right)$, and $\mathbf{H}$ is symmetry, then the proposed FTC can be used. And the numerical simulations contain two part, the first part is to verify the effectiveness of propose FTC for the non-affine dynamic system with actuation failures, these results are shown in Figure 3 - Figure 5.

The initial condition is $\mathbf{x} 0=[3,2]^{T}$, and the parameters are $k_{0}=5, k_{1}=5, a_{1}=1.5$, $a_{2}=1.5, \hat{\delta}(0)=0, \tau=0.1$. 
Figure 3 give the profile of the trajectories of $x_{1}$ and $x_{2}$, respectively. It can be got that the state would enter a very small neighbor area of origin, and the error is bounded, furthermore, the error are very small. Figure 4 shows the designed input signals, and they are almost smooth. Figure 5 shows the real input signals with failures described as before, it is got that, due to the random failures, the real input would be discontinuous in some times.

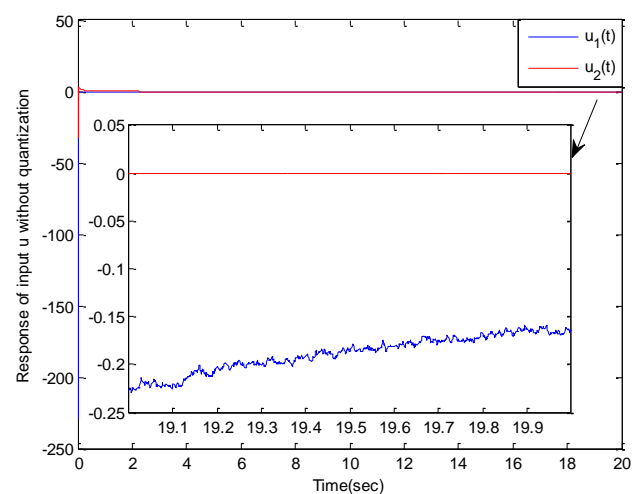

Figure4. The Designed Input Signals

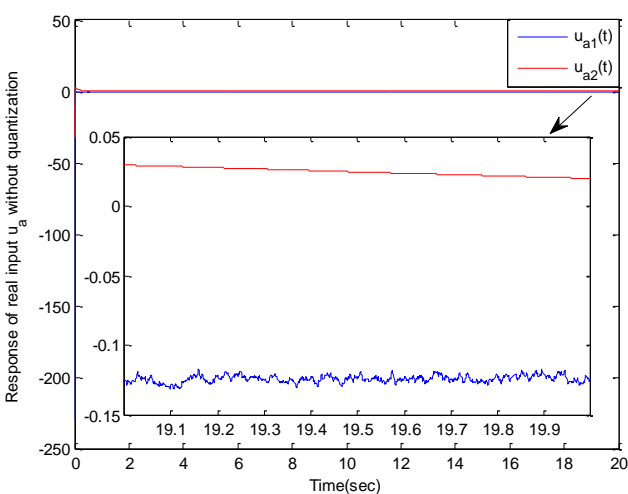

Figure 5. The Real Input Signals

\section{Conclusion}

This work exploited the control problem for a class of MIMO non-affine uncertain systems with uncertainties, disturbances, actuator failures. An adaptive robust FTC is proposed to compensate all the uncertainties, disturbances, actuator failures, and a Lyapunov-like method is proposed to guarantee that the state would be stable and ultimately uniformly bounded. Finally, a numerical simulation was presented to show the utility of the proposed adaptive robust FTC scheme.

\section{Acknowledgements}

This work was supported in part by the Education Commission of Chongqing under grant KJ1502501 "Fault tolerant control design and simulation of the power grids under sensor failures".

\section{Conflict of Interest}

The author confirms that this article content has no conflict of interest.

\section{References}

[1] J. J. E. Slotine and W. P. Li, “Applied Nonlinear Control”, Prentice Hall, Englewood Cliffs, NJ.(1991).

[2] B. Blanke. et al, "Fault-Tolerant Control Systems-A History Review", Control Eng. Pract., vol.5, no. 5,(1997),pp.693-702.

[3] J. Jian, "Fault-Tolerant Control Systems: An Introductory Overview", Acta Auto-Matica Sinica, vol. 31, (2005), pp.161-74.

[4] X. Zhang, T. Parisini and M.M. Polycarpou, "Adaptive Fault-Tolerant Control Of Nonlinear Uncertain Systems: An Diagnostic Information-Based Approach”, IEEE Transactions on Automatic Control, vol. 8, no. 49,(2004), pp.1259-1274.

[5] Z. Hu, Z. Han and Z. Tian, "Fault Detection and Diagnosis for Singular Stochastic Systems Via BSpline Expansions". ISA Transactions, vol. 48,( 2009), pp.519-524.

[6] X.D. Tang, G. Tao and S. M Joshi, "Adaptive Actuator Failure Compensation for Nonlinear Mimo Systems With an Aircraft Control Application”, Automatica, vol 11, no. 43,(2007),pp. 1869-1883. 
[7] C. Wenchuan, L. Xiaohong and S. Yongduan, "Indirect Robust Adaptive Fault-tolerant Control for Attitude Tracking of Spacecraft", Journal of Guidance, Control and Dynamics, vol. 5, no.31,( 2008), pp.1456-1463.

[8] L. Cao, X. Chen and A. K. Misra, "Minimum Sliding Mode Error Feedback Control for Fault Tolerant Reconfigurable Satellite Formations With J2 Perturbations", Acta Astronautica, vol.96, (2014),pp.201216.

[9] T. Wang, W.F. and Y.M. Zhang,"Sliding Mode Fault Tolerant Control Dealing With Modeling Uncertainties And Actuator Faults", ISA Transactions, vol. 51,(2012),pp.386-392.

[10] W. Limin, C. Xi, and G. Furong, "An LMI Method to Robust Iterative Learning Fault-tolerant Guaranteed Cost Control for Batch Processes", Chinese Journal of Chemical Engineering, vol. 4, no. 21,(2013), pp.401-411.

[11] Z. Mao, B.Jiang and P. Shi, "Observer Based Fault-Tolerant Control for A Class of Nonlinear Networked Control Systems", Journal of the Franklin Institute, vol. 347,(2010),pp.940-956.

[12] H. K. Khalil, "Nonlinear Sytems", Prentice-Hall, $3^{\text {rd }}$ edition, ( 2002).

\section{Authors}

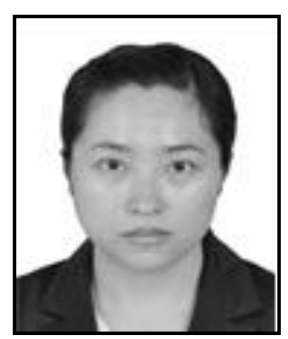

Wanqin Xiang, She received a master degree in Electric Power Systems and Automation from North China Electric Power University, China, in 2006. She is currently a lecture with Chongqing Electric Power College. Her research interests include fault diagnose and isolation, power system control.

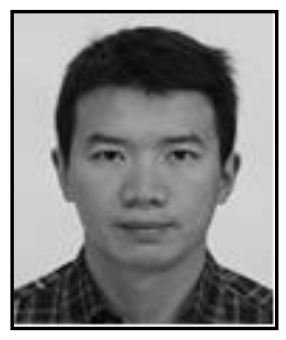

Wei Ao, He received a master degree in Automation and Engineering from National University of Defense Technology, China, in 2005. He is currently a lecture with Chongqing Communication College. His research interests include adaptive control, learning control, fault tolerant control.

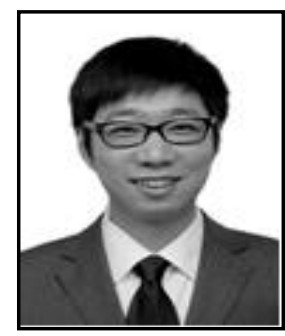

YiYuan Chen. He received a master degree in Electric Power Systems and Automation from Chongqing University, China, in 2013. $\mathrm{He}$ is currently a lecture with Chongqing Electric Power College. His research interests include fault diagnose and isolation, power system control 